\title{
Analysis of the Fe $x$ and Fe xIV line width in the solar corona using LASCO-C1 spectral data
}

\author{
M. Mierla ${ }^{1,2}$, R. Schwenn ${ }^{1}$, L. Teriaca ${ }^{1}$, G. Stenborg ${ }^{3}$, and B. Podlipnik ${ }^{1}$ \\ 1 Max-Planck-Institut für Sonnensystemforschung 37191 Katlenburg-Lindau, Germany \\ e-mail: m_mierla@yahoo.co.uk \\ 2 Astronomical Institute of the Romanian Academy, Bucharest, Romania \\ 3 Catholic University of America, Washington DC 20064, USA
}

Received 21 July 2007 / Accepted 20 November 2007

\section{ABSTRACT}

\begin{abstract}
Aims. The purpose of this paper is to analyze the variation in the line width with height in the inner corona (region above $1.1 R_{\odot}$ ), by using the spectral data from LASCO-C1 aboard SOHO. We used data acquired at activity minimum (August-October 1996) and during the ascending phase of the solar cycle (March 1998).

Methods. Series of images acquired at different wavelengths across the Fe X $637.6 \mathrm{~nm}$ (red) and Fe XIV $530.3 \mathrm{~nm}$ (green) coronal lines by LASCO-C1 allowed us to build radiance and width maps of the off-limb solar corona.

Results. In 1996, the line width of Fe XIV was roughly constant or increased with height up to around $1.3 R_{\odot}$ and then it decreased. The Fe X line width increased with height up to the point where the spectra were too noisy to allow line width measurements (around $1.3 R_{\odot}$ ). Fe X showed higher effective temperatures as compared with Fe XIV. In 1998 the line width of Fe XIV was roughly constant with height above the limb (no Fe X data available).
\end{abstract}

Key words. Sun: corona - lines: profiles - Sun: atmosphere

\section{Introduction}

Spectroscopic observations of the solar corona in emission lines help us in better understanding the dynamics of the solar corona. Emission profiles from forbidden transitions of Fe XIV $(530.3 \mathrm{~nm})$ and $\mathrm{Fe} X(637.6 \mathrm{~nm})$ contain information on physical parameters such as temperature, mass motion, and turbulence. Coronal emission lines at visible wavelengths have been observed using Fabry-Perot (FP) techniques or spectrographs during total solar eclipses and with coronagraphs at high altitudes (e.g., Singh 1985; Ichimoto et al. 1995; Hara \& Ichimoto 1999). This kind of observation is constrained by scattering effects in the terrestrial atmosphere and rarely goes up to $1.5 R_{\odot}$ (Singh et al. 2006). The LASCO (Large Angle Spectrometric Coronagraph, Brueckner et al. 1995) C1 aboard the SOHO (Solar Heliospheric Observatory) spacecraft (Domingo et al. 1995), with its field of view (FOV) covering the solar corona between 1.1 and $3 R_{\odot}$, has provided unprecedented observations of these spectral lines since its launch in December 1995 until June 1998. In the C1 design, a FP interferometer is used as a narrow-passband, tunable filter. The instrument produces a series of images that are separated in wavelength. This way, an emission line can be sampled in wavelength, generating a complete line profile simultaneously at each point in the FOV. The study of the obtained line profiles can be invaluable in a) determining the line widths and hence ion temperatures over the entire extent of the corona; b) providing unambiguous evidence of mass flows in the corona from the Doppler shift of the line (see Mierla et al. 2005).

This paper concentrates on the line width, whose measurement can provide useful information concerning the ion temperatures, turbulent motions, and sub-resolution velocity fluctuations associated with magnetohydrodynamic waves in the solar corona. The intense forbidden lines of the solar corona are magnetic dipole transitions between levels of the ground configuration of highly ionized atoms. The emissivity of the green line is due to a forbidden transition of the Fe XIV ion at $530.3 \mathrm{~nm}$ (ground configuration: $3 \mathrm{~s}^{2} 3 \mathrm{p}$ and transition: ${ }^{2} \mathrm{P}_{3 / 2}-{ }^{2} \mathrm{P}_{1 / 2}$ ). It peaks at a temperature of about 2 MK (Burgess \& Seaton 1964; Esser et al. 1995). The red coronal emission line at $637.4 \mathrm{~nm}$ (ground configuration: $3 \mathrm{~s}^{2} 3 \mathrm{p}^{5}$ and transition: ${ }^{2} \mathrm{P}_{1 / 2}-{ }^{2} \mathrm{P}_{3 / 2}$ ) from Fe $\mathrm{X}$ ions has its maximum emissivity at a temperature of around $1 \mathrm{MK}$. Below $1.2 R_{\odot}$ the mode of excitation of Fe $\mathrm{X}$ $637.4 \mathrm{~nm}$ line is predominantly collisional. Both collisional and radiative excitation (from the photospheric continuum radiation) are important for $1.2 R_{\odot}<R<1.3 R_{\odot}$, whereas radiative excitation becomes dominant beyond $R=1.3,1.4 R_{\odot}$ (Singh 1985; Raju \& Singh 1987). For Fe XIV the dominant excitation mechanism is collisional in the inner coronal regions (up to $\approx 1.4 R_{\odot}$ ) (Raju et al. 1991). For a line profile of Gaussian shape, the Doppler width, $\Delta \lambda$, is related to the standard deviation, $\sigma$, and full width at half maximum (FWHM), through

$\Delta \lambda=\sigma \sqrt{2}=F W H M /(2 \sqrt{\ln 2})$.

Assuming a Maxwellian velocity distribution of the photonemitting ions of mass $m_{1}$, a relation between $\Delta \lambda$ and the effective kinetic ion temperature $T_{\mathrm{i}}$ (comprising both thermal and subresolution non-thermal motions) can be obtained:

$\Delta \lambda=\left(\lambda_{0} / c_{0}\right) \sqrt{2 k T_{\mathrm{i}} / m_{\mathrm{i}}}=\left(\lambda_{0} / c_{0}\right) v_{1 / \mathrm{e}}$

where $k$ is the Boltzmann constant and $v_{1 / \mathrm{e}}$ is the most likely speed along the line-of-sight (LOS). If the instrumental profile 
can also be approximated by a Gaussian (of Doppler width $\phi$ ), then we can write:

$\Delta \lambda=\left(\lambda_{0} / c_{0}\right) \sqrt{2 k T_{\mathrm{i}} / m_{\mathrm{i}}+\phi^{2}}$.

\subsection{Spectroscopic observations of the solar corona}

Based on Skylab data, Doschek et al. (1977), Nicolas et al. (1977), and Mariska et al. (1978) have shown that the line width increases with height above the limb. These earlier observations were restricted to emission lines emitted by chromospheric or transition region ions formed at temperatures lower than $2.2 \times 10^{5} \mathrm{~K}$. Hassler et al. (1990), analyzing Mg X (60.9 nm and $62.5 \mathrm{~nm})$, Fe XII $(124.2 \mathrm{~nm}), \mathrm{O}$ V $(62.9 \mathrm{~nm})$, and $\mathrm{N} \mathrm{V}$ $(123.8 \mathrm{~nm}$ and $124.2 \mathrm{~nm})$ line profiles from the off-limb quiet corona, have seen an increase in the line widths with altitude up to around $0.2 R_{\odot}$ above the limb.

Wilhelm et al. (2004) used SUMER spectra of the Mg X $(62.5 \mathrm{~nm})$ line to find that the line width broadens from around $8.2 \mathrm{pm}$ to around $9.5 \mathrm{pm}$ between the limb and $220 \mathrm{Mm}$ $\left(0.33 R_{\odot}\right)$ above the limb in the equatorial corona. Harrison et al. (2002), using CDS data, report the $\mathrm{Mg}$ X (62.5 nm) line narrowing with height at altitudes above $50000 \mathrm{~km}$ in the quiet nearequatorial solar corona. The authors suggest that emission line broadening, at lower altitudes, is due to the outward propagation of undamped Alfvén waves, in open magnetic field regions with decreasing density with altitude. The narrowing at higher altitudes is interpreted as further evidence of coronal waves activity, but in closed magnetic field regions. Still in equatorial regions, Doyle et al. (1998) find an increase in the Si VIII line width with height above the limb, from about $0.026 \mathrm{~nm}$ at the limb to $0.028 \mathrm{~nm}$ at $25000 \mathrm{~km}\left(0.04 R_{\odot}\right)$ above the limb. Wilhelm et al. (2005) analyze spectra from combined CDS and SUMER observations of the relatively quiet equatorial corona above a small prominence and find no or very slight increases with altitude in the widths of emission lines from ions of several different elements. In polar regions, Banerjee et al. (1998) and Doyle et al. (1999) used SUMER spectra of Si VIII lines to observe an increase in the non-thermal velocity from $27 \mathrm{~km} \mathrm{~s}^{-1}$ at 27 arcsec above the limb to more than $46 \mathrm{~km} \mathrm{~s}^{-1}$ at $250 \operatorname{arcsec}\left(0.25 R_{\odot}\right)$ above the limb. An increase in the $\mathrm{Mg}$ X $62.5 \mathrm{~nm}$ width with height in polar coronal holes was also found by Wilhelm et al. (2004) in SUMER spectra. On the other hand, O'Shea et al. (2005), using Mg X $60.97 \mathrm{~nm}$ and $62.49 \mathrm{~nm}$ spectra from CDS, observed a decrease of the line width with height in coronal holes. They suggest that the line widths start to show a decrease in their values at the location, around $0.15 R_{\odot}$ above limb, where the dominant excitation changes from collisional to radiative. The decrease in the line width with height in the solar corona was explained by Zaqarashvili et al. (2006) by resonant energy conversion from Alfvén to acoustic waves near the region of the corona where the plasma $\beta$ approaches unity.

Chandrasekhar et al. (1991) have observed from eclipse data that the width of the green line increases up to $1.2 R_{\odot}$ and then decreases with height, while for the red line it increases with the radial distance up to $1.2 R_{\odot}$ from the Sun center. In coronal structures, the width of the red line increases with height above the limb at a rate between 0.05 and $0.26 \mathrm{pm} / \mathrm{arcsec}$, whereas the green line width in the same region decreases with height at a rate of 0.12 to $0.34 \mathrm{pm} / \operatorname{arcsec}$ (Singh et al. 1999, 2002). The authors explain this behavior as resulting from the increased mixing of hotter and cooler plasma with height. The mixed plasma at large heights will have an average temperature lower than that of the green line plasma and higher than the red line plasma at the bottom of the corona. Singh et al. (2003a) have observed an increase in the Fe X $637.4 \mathrm{~nm}$ and Fe XIII $1074.7 \mathrm{~nm}, 1079.8 \mathrm{~nm}$ line widths at the rates of $0.124 \mathrm{pm} / \mathrm{arcsec}$ and $0.029 \mathrm{pm} / \mathrm{arcsec}$, respectively, and a decrease in Fe XIV $530.3 \mathrm{~nm}$ line width at a rate of $-0.066 \mathrm{pm} / \mathrm{arcsec}$. They suggest that, if the ionization temperature of the species is higher than 1.6 MK, then the line width is decreasing with height, and if the ionization temperature is lower than 1.6 MK, then the line width is increasing with height above the limb. Singh et al. (2003b) have observed that in steady coronal structures the FWHM of the red line increases with height above the limb at a rate of around $0.102 \mathrm{pm} / \mathrm{arcsec}$ and the FWHM of the Fe XI $789.2 \mathrm{~nm}$ line also increases with height but at a lower average value: $0.055 \mathrm{pm} / \mathrm{arcsec}$. Singh et al. (2004) analyzed the data of Fe X (637.4 nm), Fe XI (789.2 nm), Fe XIII $(1074.7 \mathrm{~nm})$, and Fe XIV $(530.3 \mathrm{~nm})$ recorded with the coronagraph at Norikura Solar Observatory. They noticed that the $F W H M$ of the Fe $\mathrm{X}$ line increased with height in all coronal structures except for two, where the FWHM of the Fe X line decreased with height. The FWHM of the Fe XIV line decreased with height in almost all of the structures. From further high spectral and spatial resolution observations at Norikura, Singh et al. (2006) observe that a) the FWHM of the Fe XIV line decreases up to around $300 \operatorname{arcsec}\left(0.3 R_{\odot}\right)$ above the limb and then it remains more or less the same up to $500 \operatorname{arcsec}\left(0.5 R_{\odot}\right)$, and b) the $F W H M$ of $\mathrm{Fe} \mathrm{X}$ line increases up to $250 \operatorname{arcsec}\left(0.25 R_{\odot}\right)$ and subsequently remains constant.

\section{The instrument and the observations}

The LASCO instrument consists of three individual coronagraphs with nested FOVs. The $\mathrm{C} 1$ coronagraph observes the solar corona from 1.1 to $3.0 R_{\odot}$ and contains a FP interferometer that allows imaging the corona in different emission lines. The FP has a bandpass $(F W H M)$ of $0.07 \mathrm{~nm}$ and a tunable range of $1 \mathrm{~nm}$. Blocking filters, which are used as order sorters, have a free spectral range (interorder separation) of $3.5 \mathrm{~nm}$. The detector is a CCD camera with a nominal spatial resolution of 11.2 arcsec (pixel size of 5.6 arcsec) (Brueckner et al. 1995).

The data analyzed here are images taken in the lines of Fe XIV at $530.3 \mathrm{~nm}$ (green coronal line) and of Fe X at $637.6 \mathrm{~nm}$ (red coronal line) in two periods of time: 1996 (minimum of solar activity) and 1998 (on the rising phase of the solar cycle). The green line data of 1998 consist of 47 sets (from 28 to $30 \mathrm{March}$ ) each consisting of two scans of both the west and east limbs of the Sun. Each scan consists of 15 images at wavelengths ranging from $530.20 \mathrm{~nm}$ to $530.65 \mathrm{~nm}$ in steps of $0.03 \mathrm{~nm}$, along with 2 off-line images at wavelengths of $531.14 \mathrm{~nm}$ and $529.99 \mathrm{~nm}$ (the wavelengths are given relative to vacuum). The images were taken with a cadence of $1 \mathrm{~min}$, the exposure time being $25 \mathrm{~s}$. Thus, spectra are recorded in about $15 \mathrm{~min}$. The data of 1996 were taken between 1 August and 22 October in both the green and red lines. Each set consists of 9 spectral on-line images, ranging from $530.32 \mathrm{~nm}$ to $530.62 \mathrm{~nm}$ for Fe XIV data and from $637.43 \mathrm{~nm}$ to $637.92 \mathrm{~nm}$ for Fe X data, along with one off-line image taken at a wavelength of $531.14 \mathrm{~nm}$ (green) and $638.10 \mathrm{~nm}$ (red). In this period 1-2 data scans were taken per day, with an image cadence of $3 \mathrm{~min}$ and an exposure time of $25 \mathrm{~s}$ (green) and $16 \mathrm{~s}$ (red). In total, 97 (green) and 76 (red) data sets were recorded.

\section{Data reduction}

A detailed description of the data reduction is given in Mierla et al. (2005). To summarize, the following steps are applied. 

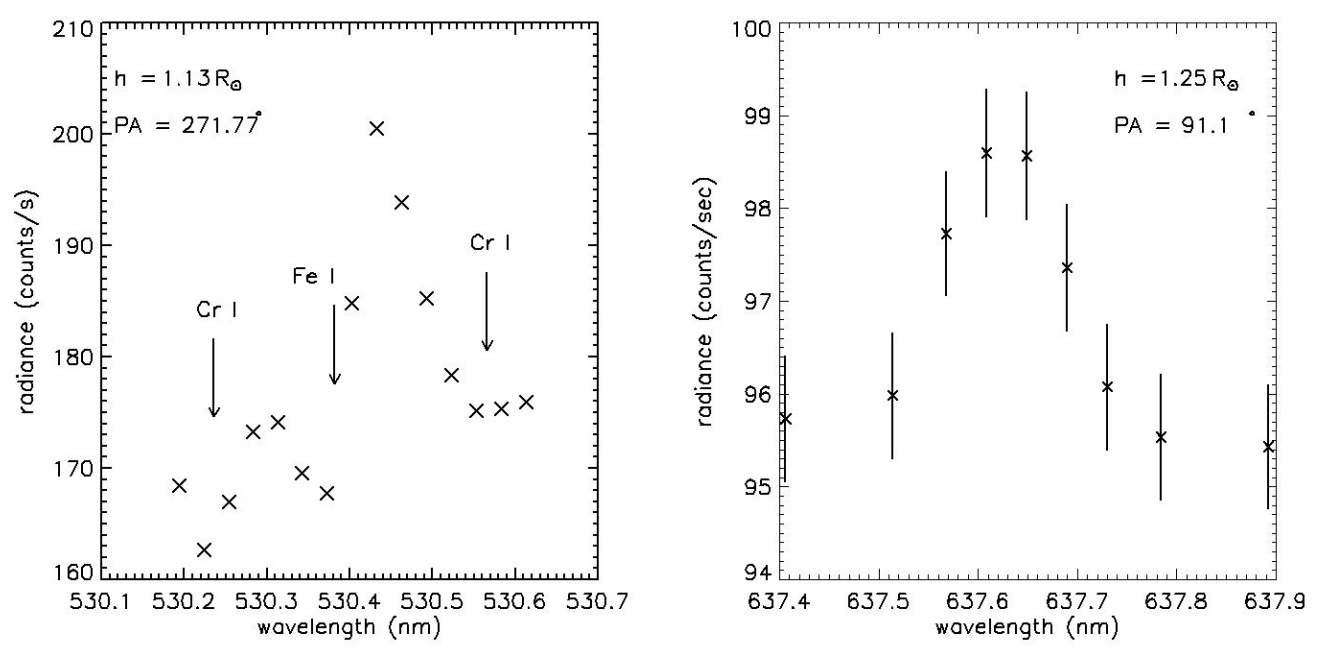

Fig. 1. Left panel: Fe XIV profile of the coronal region (PA 271 and $d=1.13 R_{\odot}$ ), as observed by LASCO-C1 on 28 March 1998. The observed profile is affected by the superimposition of the instrumental straylight photospheric spectrum comprising 3 nearby absorption line profiles (Cr I $\approx 530.23 \mathrm{~nm}$, Fe $\mathrm{I} \approx 530.37 \mathrm{~nm}$, and $\mathrm{Cr} \quad \mathrm{I} \approx 530.56 \mathrm{~nm}$ ). Right panel: $\mathrm{Fe} \mathrm{X}$ profile of a coronal region (PA 91 and $d=1.25 R_{\odot}$ ), as observed by LASCO-C1 on 20 August 1996. The errorbars were computed using Poisson distribution for the photoelectrons (one count corresponds to 13 photoelectrons). The position angle (PA) is measured counterclockwise from the north pole.
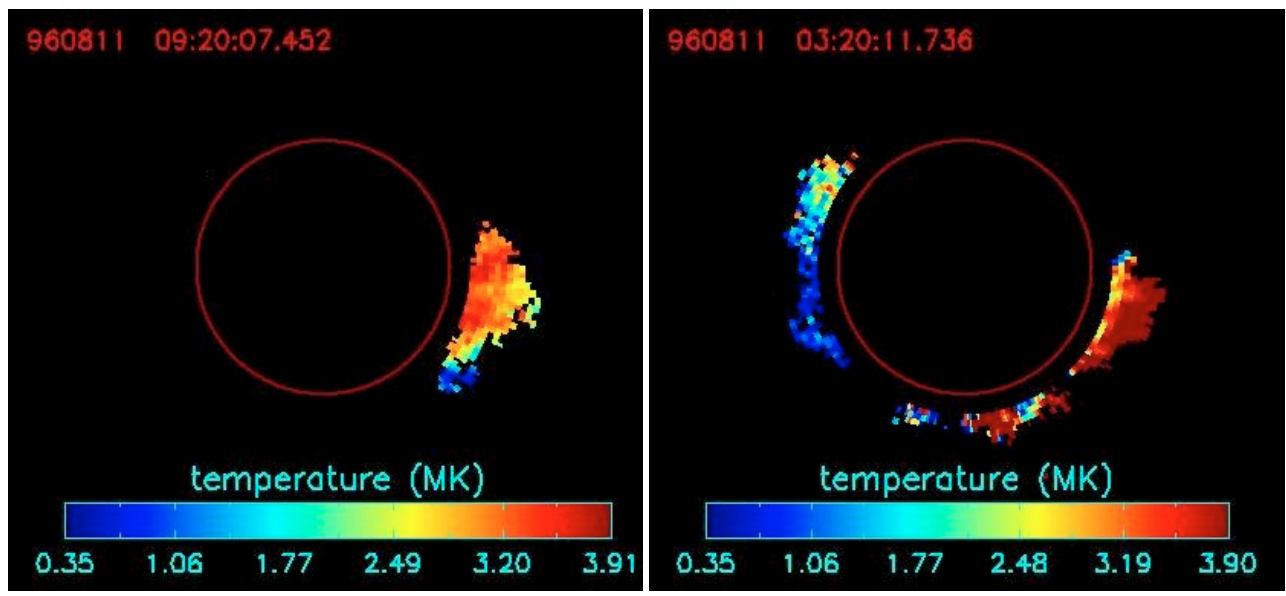

Fig. 2. Example of Fe XIV (left panel) and $\mathrm{Fe} \mathrm{X}$ (right panel) effective temperature maps for the data recorded on 11 August 1996. Larger widths (higher ion temperatures) are represented in red.

(1) In bias subtraction, the bias was added electronically on board in order to avoid negative values of the signal count rates. (2) For cosmic-ray hits removal, the corrupted values due to high energetic particles were removed by using a median filter technique. (3) For exposure time correction, the correction is only applied to Fe X data because of the smaller fraction (compared with the Fe XIV data) of count-rates due to $\mathrm{Fe} X$ photons with respect to the total signal (see Fig. 1). The emission lines are sitting on top of an intense continuum spectrum (mainly instrumental straylight). Thus, any small inaccuracy in the exposure time could affect the emission line profile when a model (errorless) background is subtracted. (4) For relative calibration of the wavelengths in the FOV of each image, the peak transmission wavelength of the FP depends on the angle of incidence that is equivalent to different spectral positions over the detector plane. (5) Absolute wavelength calibration using the main Fraunhofer line at $530.37 \mathrm{~nm}(\mathrm{Fe} \mathrm{I})$ which was observed as scattered light in the instrument (Mierla et al. 2005; Mierla 2005). This does not apply to Fe X data, because the peak of the line is far from any absorption line.

Line profiles are built by taking the radiance at a given pixel, or an ensemble of adjacent pixels (i.e., superpixel), of all images in a set as a function of the corresponding wavelengths (Fig. 1). The observed spectral profile consists of line emission from ions in the corona, photospheric light scattered at free electrons in the corona ("continuum corona"), and instrumental scattered light. The last component contains the well-known Fraunhofer lines, some of which are partially superimposed on the Fe XIV emission line profile (see left panel of Fig. 1). In the case of the $\mathrm{Fe} \mathrm{X}$ emission line, there are no relevant absorption lines at nearby wevelengths and the profile is represented well by a Gaussian (see right panel of Fig. 1). In order to improve the signal to noise ratio for the data in 1996 we created superpixels of four pixels in the radial direction and one degree (i.e., $\approx 4$ pixels at $1.1 R_{\odot}, \approx 10$ pixels at $3 R_{\odot}$ ) in the azimuthal direction. The obtained line profiles were finally fitted. For the data in 1998, we have enough signal to fit the data at each pixel of the images. The fitting procedure for Fe XIV data is described in detail by Mierla et al. (2005). For the Fe X data, a fit with one Gaussian and a constant background is performed. The FWHM maps are built by replacing each pixel (or superpixel) with the line $F W H M$ values. The line was corrected for the instrumental profile $(0.06 \mathrm{~nm}$ in the case of Fe XIV line and $0.08 \mathrm{~nm}$ in the case of Fe X; see Brueckner et al. 1995). For illustration, we show one example from data taken on 11 August 1996 (Fig. 2). An FWHM of $0.07 \mathrm{~nm}$ for Fe XIV corresponds to an effective temperature of around $2 \mathrm{MK}$, and an $F W H M$ of $0.06 \mathrm{~nm}$ for $\mathrm{Fe} \mathrm{X}$ is equivalent to $1 \mathrm{MK}$. The regions where the fit could not be performed due 

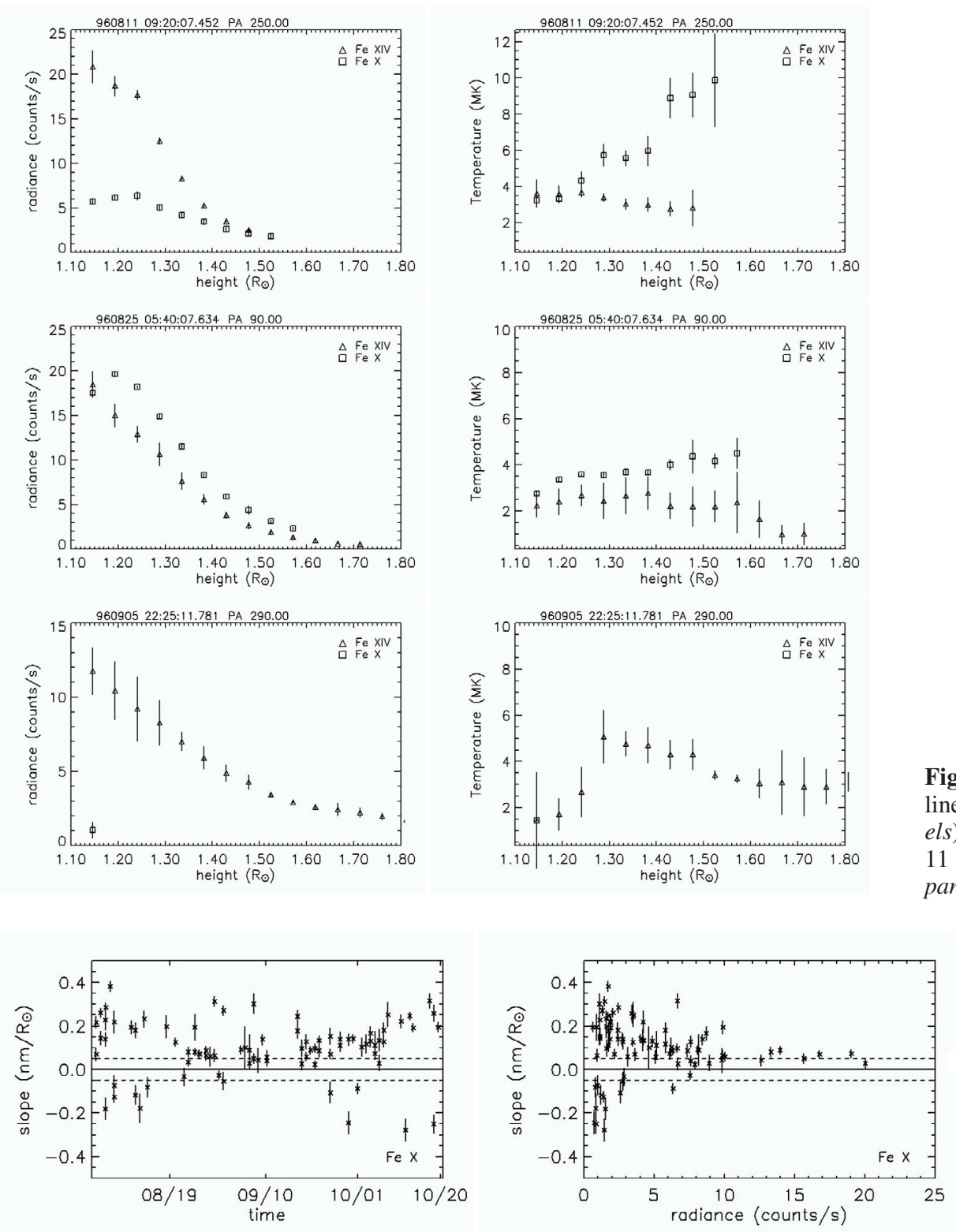

Fig. 4. Left panel: the Fe $\mathrm{X}$ slope for the data in 1996, taken at the PA: 70, 90, 110 , 250, 270, 290. The continuous horizontal line indicates a slope of zero, while the two dotted lines indicate slopes of $\pm 0.05 \mathrm{~nm} / R_{\odot}$. The $\mathrm{X}$-axis represents the date in format $\mathrm{mm} / \mathrm{dd}$. Right panel: the variation in the $\mathrm{Fe} \mathrm{X}$ slope with the radiance at $1.2 R_{\odot}$ for the same data as in left panel.

Fig. 3. Example of radial plots of the emission line radiance (left) and temperature (right panels) taken at the indicated polar angles on 11 August (upper panels), 25 August (middle panels), and 5 September (lower panels). to the weak line radiances are represented in black. In general, we can see the coronal features up to around $1.8 R_{\odot}$ with our spectral data, and reliable measurements of line widths can be performed up to $1.6 R_{\odot}$ for Fe XIV data and up to $1.3 R_{\odot}$ for Fe X data.

\section{Data interpretation}

\subsection{The line widths and the solar corona at activity minimum}

By analyzing the effective temperature maps we found that the effective ion temperatures are higher in the red corona than in the green corona, although the Fe X line has a lower formation temperature. This may mean that the amount of turbulence is higher in the cooler plasma. However, high in the corona, ions and electrons are very likely not in equilibrium, and the formation temperature (kinetic temperature of the electrons determining the ionization fraction) may not have anything to do with the ion temperature. In this context our data may indicate a preferential heating of the less ionized species (lower $q / \mathrm{m}$ ) as required by ion-ciclotron dissipation of Alfvén waves (Tu et al. 1998). From the data taken on 11 and 25 August 1996 (upper and middle panels of Fig. 3), at polar angles (PA) 250 and 90, respectively, we notice that the Fe XIV line width is roughly constant up to around $1.3 R_{\odot}$ and then decreases up to $1.7 R_{\odot}$. An increase in the line width with height is clearly visible for Fe $\mathrm{X}$ data. On 5 September (lower panels of Fig. 3), at PA 290 an increase is observed in the Fe XIV line width with height up to around $1.3 R_{\odot}$ and then a decrease up to around $1.8 R_{\odot}$. The Fe $\mathrm{X}$ data are not observed at this polar angle because of the low count rates.

\subsection{The FWHM dependence of height for green and red line data}

To analyze the radial profile of the line widths, we make radial cuts at a given PA. We apply linear fits to the measured line widths and determine the slope. For each data set, we make cuts at 6 different polar angles. We plot the slopes of all data sets versus time. We excluded the points where the correlation coefficient is between -0.5 and 0.5 . For Fe $\mathrm{X}$ data in 1996, we plot the slope in time for PA: 70, 90, 110, 250, 270, 290 as shown in Fig. 4, left panel. We see that, for the majority of the points, the 



Fig. 5. Upper panels: the variation in the Fe XIV slope with time for the data in 1996. The upperleft panel shows the slopes for the data between 1.1 and $1.3 R_{\odot}$ and the upper-right panel data between 1.3 and $1.5 R_{\odot}$. The slopes are calculated for the data taken at the PA: 70, 90, $110,250,270,290$. On the $X$-axis is represented the date in format $\mathrm{mm} / \mathrm{dd}$. Lower panels: the variation of Fe XIV slope with the radiance at $1.2 R_{\odot}$ (lower-left panel) and $1.4 R_{\odot}$ (lowerright panel) for the same data as in the above panels.
Fig. 6. Fe XIV (upper panels) maps of radiance and $F W H M$ for the data recorded on 28 of March 1998. The radial plots of the emission line parameters (middle and bottom panels) were taken at the indicated polar angles. slope is positive, i.e., the Fe $\mathrm{X} F W H M$ is increasing with radial distance throughout the entire time interval. Only in a few cases is the line width decreasing with radial distance.

Inspection of the data leads us to the suspicion that the $F W H M$ of Fe X may be affected by low count rates. To study this we plot the slope as function of line radiance for a constant radial distance of $1.2 R_{\odot}$. The result is shown in the right panel of Fig. 4. We observe that the few negative slopes generally correspond to low radiances. For Fe XIV data, we first plot the points in the range 1.1 to $1.3 R_{\odot}$ (Fig. 5, upper left panel) and then 1.3 to 

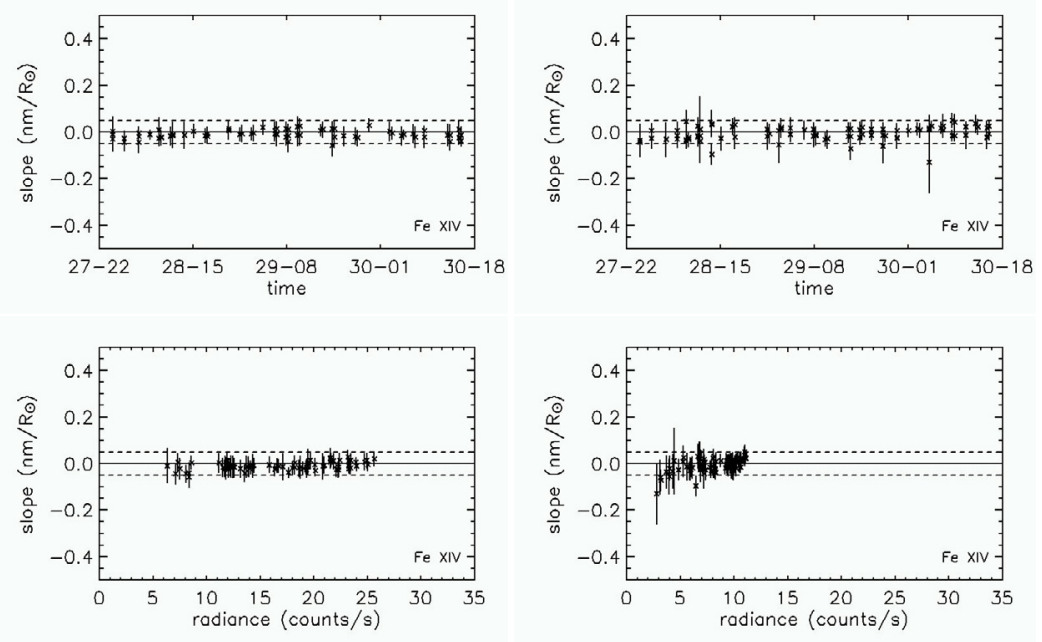

Fig. 7. Upper panels: the variation in Fe XIV slope with time for the data in 1998. The upperleft panel shows the slopes for the data between 1.1 and $1.3 R_{\odot}$ and the upper-right panel, data between 1.3 and $1.5 R_{\odot}$. The slopes are calculated for the data taken at the PA: 216, 250, 270,300 . On the $\mathrm{X}$-axis the date is represented in format dd-hh. Lower panels: the variation in Fe XIV slope with the radiance at $1.2 R_{\odot}$ (lowerleft panel) and $1.4 R_{\odot}$ (lower-right panel) for the same data as in the above panels.
$1.5 R_{\odot}$ (Fig. 5, upper right panel). We notice that, up to $1.3 R_{\odot}$, the slope is generally positive; i.e., there is almost at all times an increase in the Fe XIV FWHM with height. For larger distances, however, the Fe XIV FWHM appears to decrease with height. The slope of Fe XIV FWHM versus radiance is shown in the lower panels of Fig. 5.

\subsection{The line widths and the solar corona on the ascending phase of the solar cycle}

From Fig. 6 we find that the radiance in the active region (AR) (lower left panel) is 5 times higher than the radiance in the nonactive area (middle left panel). The steep decrease of the radiance with height at PA 215 is due to the fact that the feature is not radial and the cut just passes through a part of it. In both cases a slight decrease of the FWHM with distance is observed.

For Fe XIV data in 1998 we plot the slope (as described in Sect. 4.2) in time for PA: 216, 250, 270, 300 as shown in Fig. 7, upper panels. We see that for the majority of the points the slope shows almost no trend, i.e. the width is almost constant with radial distance (see Fig. 7). The lower panels of Fig. 7 shows the slope versus radiance.

\section{Summary}

Line profiles observed with the LASCO-C1 instrument imply ion temperatures much higher than the line formation temperature. This means that, besides thermal motions, nonthermal effects, such as turbulence, may be involved or else ions are heated to much higher temperatures than electrons. As mentioned in the introduction, there is debate regarding the variation in the line width with height. Some authors have observed a decrease in the line width with height above the solar limb and some found an increase. One possible explanation for the increase in the line width with height is the outward propagation of undamped Alfvén waves and for line width decrease with height is the resonant energy conversion from Alfvén to acoustic waves. From the data we have analyzed here we could see that, (1) in general, we see different features in red and green line images, (2) the red solar corona in 1996 was "hotter" than the green solar corona in the same period. That means that the amount of turbulence was higher in the cooler plasma or that there was preferential heating of the Fe X ions, pointing to ion-cyclotron heating. (3) For the data on 1996, the line width of Fe XIV is roughly constant or increases with height up to around $1.3 R_{\odot}$ and then it decreases to around $1.5 R_{\odot}$. The Fe $\mathrm{X}$ line width increases with height up to around $1.3 R_{\odot}$. For the data in 1998 the line width of Fe XIV is roughly constant with height above the limb. No Fe X data were available on that period. (4) The effective temperatures are much higher than than those temperatures at which Fe XIV and Fe $\mathrm{X}$ ions form (around $2 \mathrm{MK}$ and $1 \mathrm{MK}$, respectively). The excess is due to non-thermal motions (like turbulences, wave motions, etc.), LOS effects, and/or ion temperatures much higher than electron temperatures.

Acknowledgements. M.M. is thankful to Max Planck Institut für Sonnensystemforschung for the facilities to carry out this work. We are grateful to E. Marsch and R. Mecheri for productive discussions. We would like to thank the SOHO/LASCO/EIT consortia for providing the data and the software libraries. SOHO is a project of international cooperation between ESA and NASA. We also thank the anonymous referee for useful comments and suggestions.

\section{References}

Banerjee, D., Teriaca, L., Doyle, J. G., \& Wilhelm, K. 1998, A\&A, 339, 208 Brueckner, G. E., Howard, R. A., Koomen, M. J., et al. 1995, Sol. Phys., 162, 357

Chandrasekhar, T., Desai, J. N., Ashok, N. M., \& Pasachoff, J. M. 1991, Sol. Phys., 131, 25

Domingo, V., Fleck, B., \& Poland, A. I. 1995, Sol. Phys., 162, 1

Doschek, G. A., Cohen, L., \& Feldman, U. 1977, ApJS, 33, 101

Doyle, J. G., Banerjee, D., \& Perez, M. E. 1998, Sol. Phys., 181, 51

Doyle, J. G., Teriaca, L., \& Banerjee, D. 1999, A\&A, 349, 956

Hara, H., \& Ichimoto, K. 1999, ApJ, 513, 969

Harrison, R. A., Hood, A. W., \& Pike, C. D. 2002, A\&A, 392, 319

Hassler, D. M., Rottman, G. J., Shoub, E. C., \& Holzer, T. E. 1990, ApJ, 348, 77

Ichimoto, K., Hara, H., Takeda, A., et al. 1995, ApJ, 445, 978

Mariska, J. T., Feldman, U., \& Doschek, G. A. 1978, ApJ, 226, 698

Mierla, M. 2005, On the Dynamics of the Solar Corona, Ph.D. Thesis, Copernicus $\mathrm{GmbH}$

Mierla, M., Schwenn, R., Teriaca, L., Stenborg, G., \& Podlipnik, B. 2005, JASR, 35,2199

Nicolas, K. R., Brueckner, G. E., Tousey, R., et al. 1977, Sol. Phys., 55, 305

O'Shea, E., Banerjee, D., \& Doyle, J. G. 2005, A\&A, 436, 35

Raju, P. K., \& Singh, J. 1987, Sol. Phys., 110, 271

Raju, K. P., Desai, J. N., Chandrasekhar, T., \& Ashok, N. M. 1991, JApA, 12, 311

Singh, J. 1985, Sol. Phys., 95, 253

Singh, J., Ichimoto, K., Imai, H., et al. 1999, PASJ, 51, 269

Singh, J., Sakurai, T., Ichimoto, K., \& Takeda, A. 2002, PASJ, 54, 807

Singh, J., Ichimoto, K., Sakurai, T., \& Muneer, S. 2003a, ApJ, 585, 516

Singh, J., Sakurai, T., Ichimoto, K., \& Muneer, S. 2003b, Sol. Phys., 212, 343

Singh, J., Sakurai, T., Ichimoto, K., \& Watanabe, T. 2004, ApJ, 617, 81

Singh, J., Sakurai, T., Ichimoto, K. 2006, ApJ, 639, 475

Tu, C.-Y., Marsch, E., Wilhelm, K., \& Curdt, W. 1998, ApJ, 503, 475 Zaqarashvili, T. V., Oliver, R., \& Ballester, J. L. 2006, A\&A, 456, 13 Wilhelm, K., Dwivedi, B. N., \& Teriaca, L. 2004, A\&A, 415, 1133 Wilhelm, K., Fludra, A., Teriaca, L., et al. 2005, A\&A, 435, 733 